\title{
Estrategias de intervención en áreas urbanas inundables: el caso Bella Unión, Uruguay
}

Adriana Piperno. Universidad de La República, Montevideo, Uruguay

Pablo Sierra. Universidad de La República, Montevideo, Uruguay

RESUMEN | El objetivo de este trabajo es caracterizar y dar elementos para la acción en las áreas inundables de la ciudad de Bella Unión. Si bien las inundaciones no generan una situación de desastre, afectan fuertemente la economía local, impactando sobre sectores muy vulnerables de la población. El área inundable se ha visto incrementada a partir de 1979 por la puesta en funcionamiento de la represa de Salto Grande. Las acciones de indemnización se presentan como una oportunidad para realizar estudios pormenorizados de la zona y definir acciones convergentes e integrales para gestionar estas áreas. Se desarrolla una metodología basada en el análisis de la interacción del sistema hídrico con el sistema territorial, que permite reconocer los distintos niveles de riesgo de la zona afectada y los impactos asociados, considerando tanto el nivel de la amenaza como la vulnerabilidad de sus pobladores. El análisis incorpora igualmente las potencialidades de la zona, de forma de incorporar el río al imaginario colectivo de la ciudad.

PALABRAS CLAVE | riesgos naturales, planificación urbana, vulnerabilidad.

ABSTRACT | This study aims to characterize and provide items to operate in flooded areas of Bella Union city. Although floodings cannot be defined as disasters, they strongly affect the local economy, as they have an impact on vulnerable sectors of the population. Its floodplain has increased since 1979 by the installation of Salto Grande dam. Compensation actions are presented as an opportunity for developing detailed studies of the area and defining further convergent and integrated actions to manage these areas. The methodology is based on the analysis of the interaction of water and territorial systems, recognizing different levels of risk in the affected area and the associated impacts. It considers both the level of hazard and vulnerability of its inhabitants. It also recognizes the potential of the area to incorporate the river to the city's collective imaginary.

KEY WORDS | natural risks, urban planning, vulnerability

Recibido el 15 de marzo de 2011, aprobado el 19 de julio de 2011

E-mail: Adriana Piperno, apiperno@farq.edu.uy | Pablo Sierra, psierra@farq.edu.uy

Los autores agradecen sus colaboraciones en el artículo a Manuela Abrahan, Lucía Anzalone, Amancay Matos, Laura Olmedo, Virginia Osimani, Osvaldo Sabaño. Participaron en el equipo de investigación: Pablo Antonaz, Carlos Castro, Andrés Dibarboure, Amancay Matos, Rafael Tornini. 


\section{Introducción}

Bella Unión, la ciudad más alejada de la capital, ubicada en la triple frontera entre Uruguay, Brasil y Argentina, cuenta con 13.187 habitantes (censo de 2004) y es la segunda ciudad del departamento de Artigas, uno de los más pobres del país. Situada sobre la margen oriental del río Uruguay, es uno de los centros poblados con problemas de inundación recurrentes. Si bien las mismas no pueden ser catalogadas de desastrosas, afectan fuertemente la economía local, impactando sobre sectores muy vulnerables de la población.

En 1979 se construyó aguas abajo de la ciudad la represa hidroeléctrica binacional de Salto Grande. El represamiento del río generó un embalse cuyo manejo impacta sobre la ciudad de Bella Unión, aumentando el área inundable y la probabilidad de afectación de los eventos en la misma.

La Ley No 15.845 del 15 de diciembre de 1986 dispone que el Estado es civilmente responsable de los eventuales daños ocasionados por el impacto de la presa, por lo cual debe indemnizar a aquellos inmuebles expuestos a las crecidas temporarias provocadas por esta. A los efectos de resolver este relegado problema, en mayo de 2010 el Ministerio de Economía y Finanzas (MEF) firmó un convenio con la Universidad de la República (UdelaR), para ser asesorado en la definición de criterios para la resolución del mismo.

Paralelamente, el gobierno nacional y local priorizan para este quinquenio, 20102014, una política de vivienda focalizada hacia sectores vulnerables en áreas inundadas y contaminadas y a la realización de los planes locales de las ciudades, a partir del mandato de la Ley de Ordenamiento Territorial y Desarrollo Sostenible (Ley No 13.808) aprobada en setiembre de 2009. La convergencia de prioridades en este territorio ha generado un contexto propicio para definir estrategias interinstitucionales.

Por otra parte, la resolución de este problema permite verificar, ajustar y profundizar instrumentos metodológicos desarrollados en instancias anteriores por el equipo de investigación para la evaluación del daño y diseño de estrategias de recuperación en las ciudades afectadas por los eventos de inundación (Piperno \& Sierra, 2009; Piperno, Sierra, Varela \& Failache, 2009), llevadas a la práctica durante los eventos de 2007, 2009 y 2010, en coordinación con el Sistema Nacional de Emergencias (Instituto de Teoría y Urbanismo [ITU], 2007; Grupo de Gestión Integral del Riesgo [GGIR], 2010).

En este artículo se reflexiona en torno a la multidimensionalidad del abordaje del problema señalado, la integración de diferentes saberes disciplinares y la profundización en las metodologías de trabajo.

La estrategia metodológica se basa en el análisis de la interacción del sistema hídrico con el sistema territorial, de manera de reconocer los distintos niveles de riesgo de la zona afectada, considerando tanto el nivel de la amenaza como la vulnerabilidad de sus pobladores. Asimismo, se reconocen las potencialidades de la zona en cuanto a la forma de incorporar el río al imaginario colectivo de la ciudad.

En síntesis, se caracterizan las áreas urbanas inundables en la ciudad de Bella Unión y su microrregión (en particular Pueblo Cuareim), dando elementos para 
actuaciones integrales en las mismas dirigidas a la mitigación del impacto de los eventos de inundación.

\section{Bases conceptuales y metodológicas}

El riesgo es la probabilidad de la ocurrencia de algo nocivo para un grupo humano o sociedad determinada. Esto implica la existencia de un "peligro" o "amenaza" y una población afectada por el mismo.

En palabras de Lavell (1996), el riesgo debe ser reconocido como "una condición latente o potencial", cuyo "grado depende de la intensidad probable de la amenaza y los niveles de vulnerabilidad existentes" (p. 10). Se entiende la amenaza o peligrosidad como "el potencial peligroso que tienen los fenómenos naturales (espontáneos o manipulados técnicamente), potencial inherente al fenómeno mismo, sea cual sea el grado de artificialidad" (Barrenechea, Gentile, González \& Natenzon, 2000, p. 2); en la vulnerabilidad se identifica la exposición como la "condición de susceptibilidad que tiene el asentamiento humano de ser afectado por estar en el área de influencia de los fenómenos peligrosos y por su fragilidad física"; y la fragilidad social se define como la "predisposición que surge como resultado del nivel de marginalidad y segregación social del asentamiento humano y sus condiciones de desventaja y debilidad relativa por factores socioeconómicos" (Cardona, 2001, p. 106).

En esta perspectiva, se traslada el centro de la problemática desde la identificación del evento concreto como principal "responsable" del desastre, hacia el reconocimiento del riesgo como un proceso. Por lo tanto, en la acción frente al desastre se incluye otros momentos, además de la actuación específica en la emergencia. Gestionar el riesgo implica, entonces, la adopción de políticas, estrategias y prácticas (físicas, culturales, institucionales, económicas, etcétera) orientadas a reducir los riesgos de desastres o minimizar sus efectos. Tales medidas deben ser analizadas e implementadas como parte de los procesos de desarrollo, ya que "todo proceso de desarrollo, de transformación territorial y ambiental debe ser informado por un proceso de análisis y control sobre los factores de riesgo existentes o posibles" (Lavell, 2002, p. 2).

Para integrar el riesgo en la planificación Cardona (2003) involucra tres políticas públicas: la identificación del riesgo (representación individual, representación social y estimación objetiva), la reducción del riesgo (prevención - mitigación) y el manejo de desastres (respuesta y recuperación).

En el caso de Uruguay, la magnitud de los desastres —entendidos como "interrupción seria del funcionamiento de una comunidad o sociedad que causa pérdidas humanas y/o importantes pérdidas materiales, económicas o ambientales; que exceden la capacidad de la comunidad o sociedad afectada para hacer frente a la situación utilizando sus propios recursos" (Estrategia Internacional para la Reducción de Desastres Las Américas [EIRD], 2004) - es relativamente menor en cuanto a número de víctimas y damnificados. Sin embargo, su recurrencia en comunidades 
locales con baja resistencia y resiliencia ${ }^{1}$ a este tipo de eventos magnifica su impacto, constituyéndose en verdaderas trabas a los procesos de desarrollo local. Se pone en evidencia de esta manera la íntima relación existente entre la gestión de los riesgos y las políticas de desarrollo, en particular teniendo en cuenta que muchas veces son los propios procesos de desarrollo los que contribuyen a propiciar situaciones de riesgo.

Gestionar esos "desastres de pequeña escala" como los que ocurren en Uruguay genera la necesidad de reformular las metodologías y procedimientos propios de la gestión de eventos de gran magnitud. En este sentido, se torna primordial identificar y potenciar aquellas particularidades que permiten, entre otros aspectos, un trabajo muy próximo a los implicados, con un conocimiento "cara a cara" que habilita procesos participativos en la definición de las estrategias de gestión del riesgo. Debe considerarse, al mismo tiempo, que el monto de las inversiones necesarias para prevenir y mitigar estos eventos de pequeña escala es menor y, por lo tanto, posible de ser considerado en los presupuestos de las diferentes instituciones involucradas.

El trabajo que aquí se presenta propone potenciar las oportunidades que ofrece el proceso de gestión de riesgos de pequeña escala y dar elementos para replicarloen otras realidades similares del país. Su punto de partida es el análisis del ecotono ${ }^{2}$ río-ciudad como la interacción del sistema hídrico y el sistema territorial. A partir del primero se caracteriza la "amenaza"; y a partir del segundo, la "vulnerabilidad", en sus dos aspectos: exposición y fragilidad. El riesgo es función de esta interacción, y a partir de su caracterización se definen los principales lineamientos de acción.

\section{Sistema hídrico}

Para realizar un análisis del riesgo, se identifican en primera instancia los niveles de peligro, a partir de los cuales se delimita la zona de estudio.

La definición del límite de la zona inundable, entregada como insumo al equipo de investigación, se realizó a partir del nivel alcanzado por el agua en el máximo evento registrado después de construida la represa, correspondiente a julio de 1983, donde se evacuó a 913 personas. A partir de dicho límite, registrado en campo por la Intendencia Departamental de Artigas (IdeA), se propuso una "zona de servidumbre”, que se adoptó como primera aproximación al área de estudio.

El impacto de la amenaza no es homogéneo en la zona inundable, por lo que es necesario territorializarlo. Ello se realiza a partir de dos elementos: la frecuencia de inundación según periodos de retorno, obtenidos sobre la base del modelo hidrodinámico; y la altura que alcanza el agua en el interior de la vivienda, ambos registrados a partir del trabajo de campo y de la altura de la vivienda en relación con la calle.

Para la determinación de la frecuencia de las inundaciones, se solicitó a la Comisión Técnica Mixta de Salto Grande (CTM) la corrida de su modelo hidrodinámico, proporcionándole para ello un relevamiento topográfico con curvas de nivel cada medio metro, generado a partir de la cartografía del Servicio Geográfico Militar, que cuenta con curvas de nivel cada dos metros.

1 Se entiende por 'resiliencia' el acceso y movilización de recursos del asentamiento humano, su capacidad de respuesta y absorción del impacto (Véase Cardona, 2001, p. 106).

2 'Ecotono' es la zona de transición natural entre dos ecosistemas distintos. 
Para determinar la profundidad del agua, se manejó además la información surgida del relevamiento de campo realizado. Las particularidades de este aspecto se desarrollarán más adelante.

\section{Modelación del río ${ }^{3}$}

El estudio se realizó a partir de los valores estadísticos de los caudales máximos registrados en el río Uruguay en Salto Grande y los resultados de un modelo hidrodinámico (que relaciona cotas con caudales) que analiza las situaciones en función de los caudales en los ríos y la cota del embalse.

Para la modelación estadística, se ajustó la distribución estadística de eventos extremos Gumbel en la serie de CTM-SG 1898-2010. De esta manera se determinaron los caudales asociados a periodos de retorno con un intervalo de confianza de $95 \%$. El Cuadro 1 presenta los valores de caudales para periodos de retorno ( $\mathrm{Tr}$ ) entre 2 y 1000 años. Se trabaja fundamentalmente con los Tr de 100, 50, 25 y 10, por ser los considerados por la Dirección Nacional de Aguas (Dinagua) del Ministerio de Vivienda, Ordenamiento Territorial y Medio Ambiente en el proyecto de Directrices Nacionales de Inundaciones de Ribera (2010).

CUADRO 1 Valores de caudales para periodos de retorno entre 2 y 1000 años

\begin{tabular}{l|lll}
\hline \multirow{2}{*}{$\begin{array}{l}\text { PERIODO DE } \\
\text { RETORNO }\end{array}$} & \multicolumn{3}{|l}{ INTERVALO DE CONFIANZA DEL $95 \%$} \\
\cline { 2 - 4 } & $\begin{array}{l}\text { INTERVALO INFERIOR } \\
\left(\mathrm{M}^{3} / \mathrm{s}\right)\end{array}$ & VALOR CENTRAL $\left(\mathrm{M}^{3} / \mathrm{s}\right)$ & $\begin{array}{l}\text { INTERVALO SUPERIOR } \\
\left(\mathrm{M}^{3} / \mathrm{s}\right)\end{array}$ \\
\hline 2 & 15.502 & 16.826 & 18.149 \\
5 & 20.800 & 22.959 & 25.118 \\
10 & 24.307 & 27.019 & 29.732 \\
25 & 28.738 & 32.150 & 35.561 \\
50 & 32.025 & 35.956 & 39.886 \\
75 & 33.936 & 38.168 & 42.400 \\
100 & 35.289 & 39.734 & 44.179 \\
1000 & 46.071 & 52.217 & 58.363 \\
\hline
\end{tabular}

fuente Comisión Técnica Mixta de Salto Grande (2011).

Una vez calculados los niveles y caudales en Salto Grande para distintos periodos de retorno, se estimaron los niveles en la ciudad de Bella Unión. Estos niveles se encuentran afectados por el de la represa de Salto Grande ya que, para un mismo caudal, dependiendo del nivel en que se encuentre la represa, se producirán distintos niveles en la ciudad de Bella Unión (curvas de remanso). Para esto se utilizó un modelo hidrodinámico ${ }^{4}$ en estado estacionario para diferentes situaciones

3 Estos apartados fueron realizados a partir de estudios elaborados por técnicos de la CTM explicitados en el Informe de Trabajo Cotas de Inundación en Bella Unión y Pueblo Cuareim (2011).

4 Se utiliza el programa unidimensional HEC-RAS desarrollado por el Cuerpo de Ingenieros del Departamento de Defensa de los Estados Unidos. 
(Gráfico 1). A los efectos del caso Bella Unión, son de particular trascendencia la cota de embalse 33,50, por debajo de la cual el informe determina que los niveles del embalse no afectan los niveles en la ciudad; y la cota 36, definida como el nivel crítico de manejo de la presa para crecidas extraordinarias. Esta información se espacializa en la Figura 1.

GRÁfico 1 Cotas de Bella Unión en función de los caudales y cotas de presa e impacto de la presa en $\operatorname{Tr}=1,10$ y 100

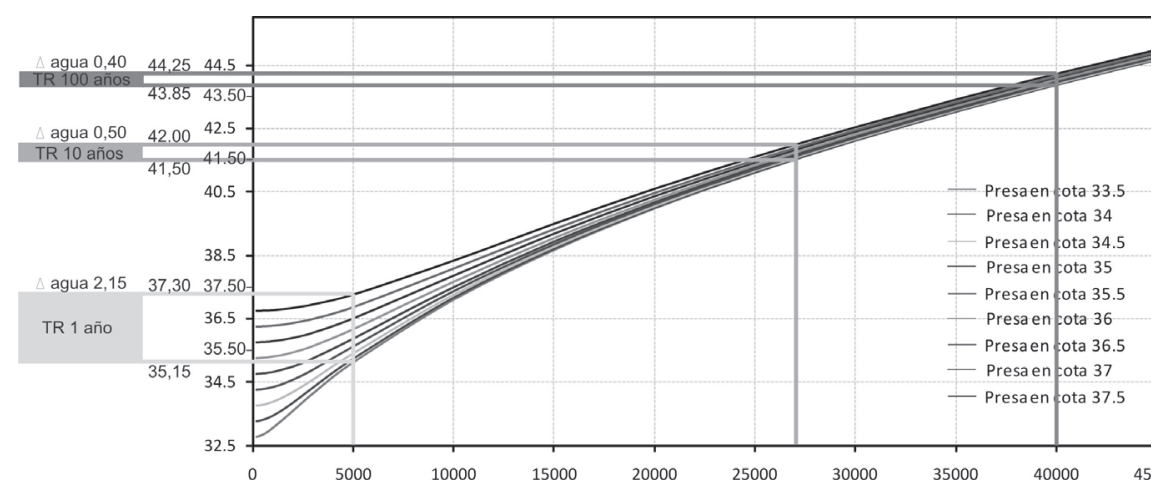

FUENTE ELABORACIÓN PROPIA A PARTIR DE GRÁFICO EN CTM (2011).

FIGURA 1 Curvas por periodo de retorno en Bella Unión

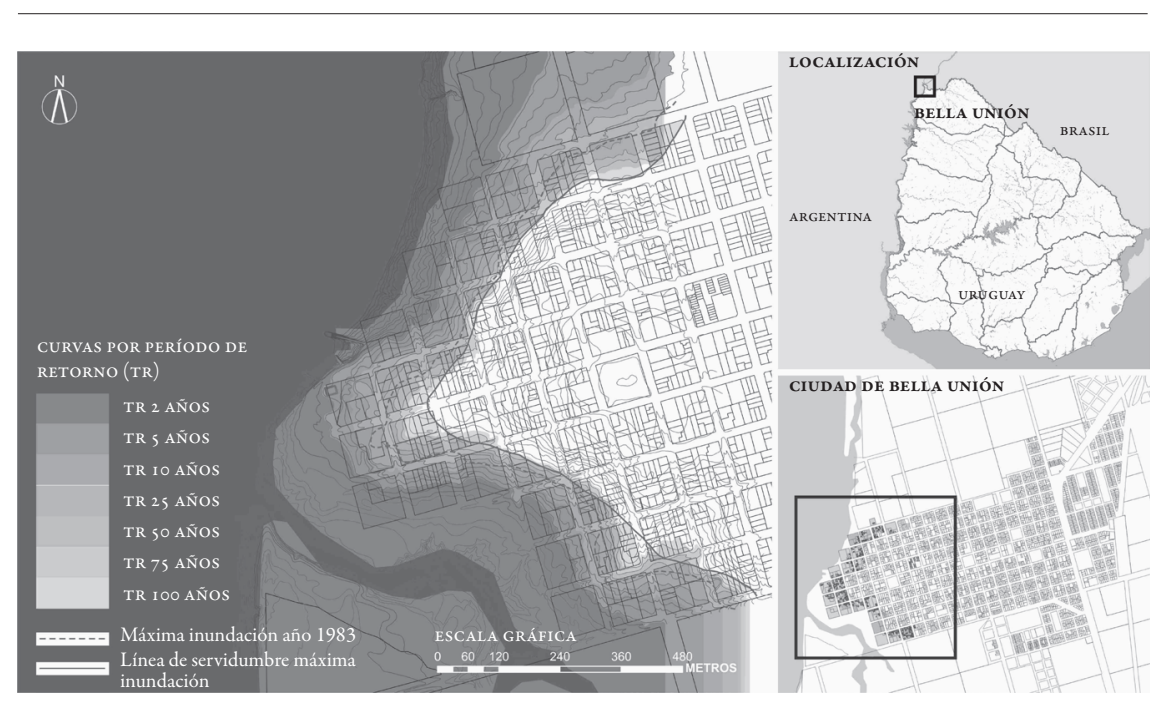

FUENTE ELABORACIÓN PROPIA. 


\section{Sistema territorial (aspectos sociales, territoriales y jurídicos)}

Para intervenir en la zona inundable, es necesario reconocer tanto las características intrínsecas de la misma (espacio público, viviendas y pobladores) como el rol que ella desempeña en el sistema urbano. Se trata de construir los insumos necesarios para realizar un análisis prospectivo de las acciones posibles de emprender; definir estrategias a corto, mediano y largo plazo; avaluarlas y evaluarlas.

Para caracterizar el sistema territorial, se realiza una triangulación de diferentes métodos y fuentes:

a) Fotointerpretación. Sirve fundamentalmente como apoyo al análisis de las dinámicas territoriales. Para este estudio se realizó a partir de la lectura de fotos aéreas de 1978, 1995 y 2004, espacializándose la ocupación de predios con relación a esas fechas.

b) Reconocimiento visual. Mediante una ficha por unidad, se relevan de manera individualizada las características de las construcciones existentes en la zona. En este caso se incorporó, además, el registro fotográfico del exterior e interior de cada unidad. Asimismo, se realizó un relevamiento a nivel general de la infraestructura y equipamientos, uso de los espacios, calidad ambiental, etcétera, a los efectos de obtener una visión general de las características del barrio.

c) Encuestas. Se aplicó la metodología diseñada por el equipo para la evaluación del daño en los eventos de inundación de 2007, 2009 y 2010. A través de la misma, se conocen las características del núcleo familiar y su percepción sobre el barrio y, específicamente, sobre el evento de inundación. Como unidades de relevamiento de la información se utilizaron el predio (que en el registro catastral se presenta con un identificador único "número de padrón"), la unidad de ocupación (asimilable por lo general a la vivienda), el sector constructivo de la unidad y la persona (Gráfico 2). Todos ellos contienen un ID que permite la relación entre la información y su georreferenciación.

d) Análisis de registros públicos. De ellos se identifica la tenencia del suelo (Registros de propiedad del Ministerio de Educación y Cultura, MEC) y los valores catastrales del bien (Dirección Nacional de Catastro, Ministerio de Economía y Finanzas).

e) Otras fuentes complementarias. Entrevistas calificadas, fotografías históricas (Figura 2), entre otras, complementan la información recabada.

Al generar y procesar la información obtenida se puso especial atención en que la misma pudiera integrarse a un Sistema de Información Nacional que potencie la información generada por diferentes usuarios, habilitando nuevos análisis. Es así que se construyó un sistema de información geográfica utilizando ArcGis y gvSIG, tomando como base la cartografía de la Infraestructura de Datos Espaciales (IDE). Cuando fue posible, las variables y la formulación de las preguntas se realizaron siguiendo criterios de organismos nacionales competentes (Instituto Nacional de Estadísticas, entre otros). 
GRÁFICO 2 Unidad de relevamiento

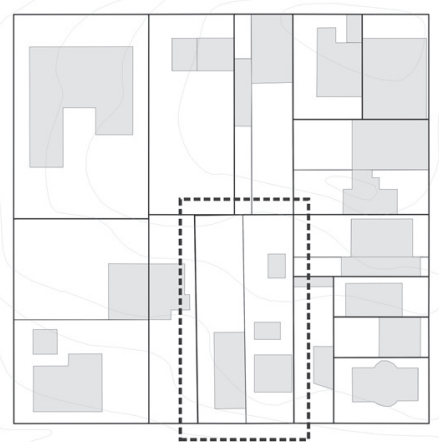

PERVIVIENDA UNIDAD 2

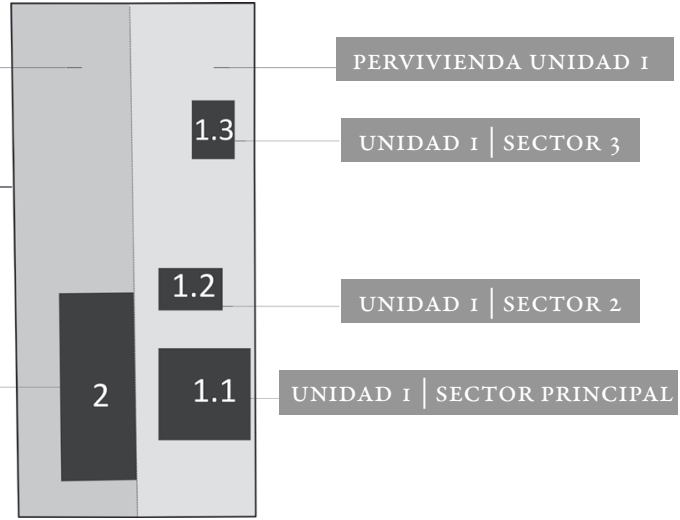

FUENTE ELABORACIÓN PROPIA.

FIGURA 2 Registros de eventos de inundación

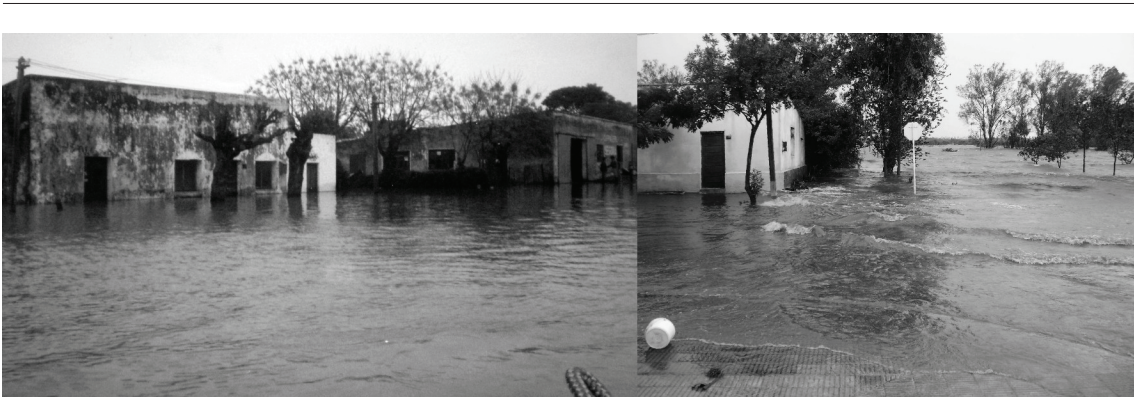

FUENTE Vecinos ReleVAdos.

\section{Análisis de la vulnerabilidad: exposición}

Más allá de la magnitud del fenómeno de la inundación, son las condiciones de vulnerabilidad de la zona las que definen el riesgo. Estas condiciones dificultan el desarrollo de la comunidad y la hacen más frágil ante eventos adversos. 
En cuanto a la exposición al fenómeno, se relevaron 415 unidades y 695 personas, ubicadas en 217 predios del área inundable de Bella Unión y Pueblo Cuareim (Cuadro 2). En este artículo se presentan los análisis correspondientes a Bella Unión.

\section{CUADRo 2 Unidades relevadas en Bella Unión y Pueblo Cuareim}

\begin{tabular}{l|lcc}
\hline \multirow{2}{*}{$\begin{array}{l}\text { PERIODO DE } \\
\text { RETORNO }\end{array}$} & \multicolumn{3}{|l}{ INTERVALO DE CONFIANZA DEL 95\% } \\
\cline { 2 - 4 } & $\begin{array}{l}\text { INTERVALO INFERIOR } \\
\left(\mathrm{M}^{3} / \mathrm{s}\right)\end{array}$ & VALOR CENTRAL $\left(\mathrm{M}^{3} / \mathrm{s}\right)$ & $\begin{array}{l}\text { lNTERVALO SUPERIOR } \\
\left(\mathrm{M}^{3} / \mathrm{s}\right)\end{array}$ \\
\hline 2 & 15.502 & 16.826 & 18.149 \\
5 & 20.800 & 22.959 & 25.118 \\
10 & 24.307 & 27.019 & 29.732 \\
25 & 28.738 & 32.150 & 35.561 \\
50 & 32.025 & 35.956 & 39.886 \\
75 & 33.936 & 38.168 & 42.400 \\
100 & 35.289 & 39.734 & 44.179 \\
1000 & 46.071 & 52.217 & 58.363 \\
\hline
\end{tabular}

FUENTE ELABORACIÓN PROPIA.

El $86 \%$ de las unidades relevadas son habitacionales, correspondiendo $76 \%$ a unidades de uso exclusivamente habitacional y $10 \%$ a casa habitación compartida con otro uso (comercios, servicios, industria, etcétera). El 5\% de las unidades desarrollan solamente actividades distintas a la habitación.

Las unidades relevadas están ocupadas en un 82\%. Las desocupadas corresponden, el $3 \%$ a viviendas en construcción, y el $7 \%$ a viviendas y locales abandonados.

La espacialización de la información da cuenta de que la zona inundable no es homogénea; presenta en su interior zonas de comportamiento diferencial que deben ser analizadas de manera independiente, a los efectos de definir estrategias de acción para las mismas.

A partir del mapeo del Índice de Vulnerabilidad Social (IVS) ${ }^{5}$, de las características del stock edilicio identificado a partir del relevamiento y de la consideración de la frecuencia del evento, se realizan las siguientes zonas que serán las unidades de análisis territorial (Figura 3):

- Zona Puerto. Sector consolidado, estructurado sobre el eje de las principales vías urbanas de la ciudad (calles Rivera y Artigas). Presenta viviendas en general buenas, que cuentan con todos los servicios. Se encuentra asociado al Parque costero, de importancia fundamental para la ciudad como espacio de recreación y por sus valores paisajísticos.

- Zona Norte. Se encuentra alejada del centro y presenta carencias de equipamientos urbanos (déficit en alumbrado público, calles sin pavimentar y, en general, sin red de saneamiento).

5 El IVS, elaborado por Dinagua, integra indicadores sociales relevados en el trabajo de campo: ocupación, clima educativo, participación, hacinamiento, estado de la vivienda, acceso a servicios básicos y percepción de la inundación. 
- Zona Sur. Es un sector con predominancia de viviendas económicas y familias asentadas hace años, con un fuerte arraigo al barrio. Presenta carencias de equipamientos urbanos, al igual que el resto de los sectores periféricos de la ciudad.

Tanto la Zona Norte como la Sur presentan sectores en los cuales las inundaciones son más frecuentes. Se caracterizan por la precariedad en el stock edilicio y en la tenencia del suelo, y por contar con los índices más altos de vulnerabilidad social. Debido a esto se las define como áreas de relocalización.

FIgURA 3 Zonas de análisis

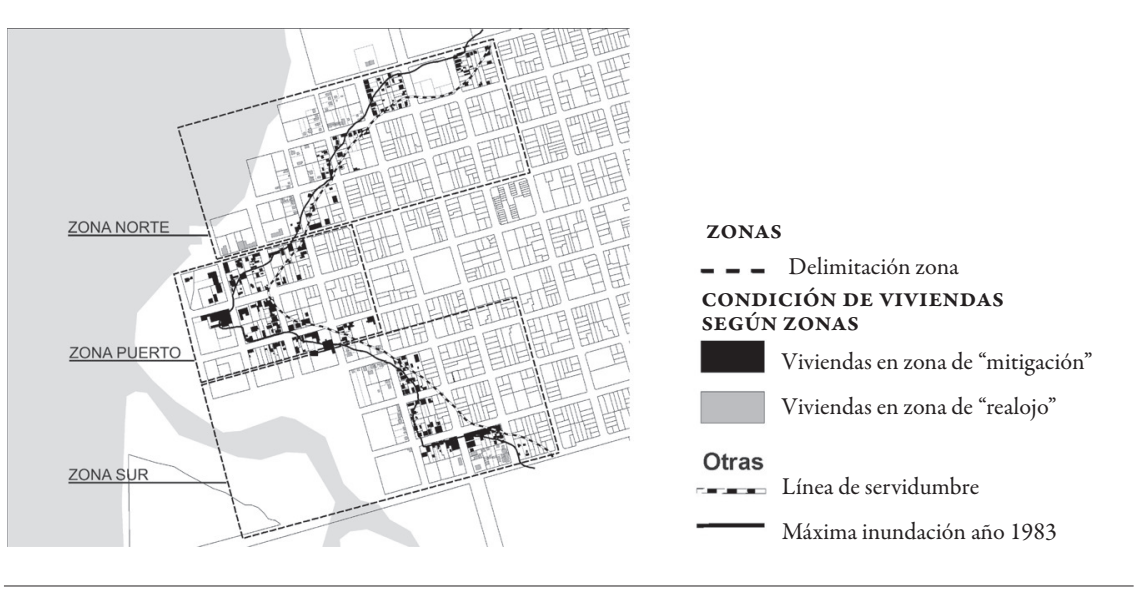

FUENTE ELABORACIÓN PROPIA.

\section{Análisis de la vulnerabilidad: fragilidad social}

Las zonas Sur y Norte se presentan como más vulnerables que la zona Puerto. Entre ellas, son más vulnerables aquellos sectores próximos al río, esto es, con mayor frecuencia de inundación. El sector Norte presenta mayor vulnerabilidad que el Sur, con menor arraigo de sus habitantes y menor integración a redes sociales. Ambos sectores presentan "comportamientos de periferia".

Para analizar en detalle la vulnerabilidad, es necesario considerar sus múltiples dimensiones. Se reconoce una vulnerabilidad física y material, en el entendido de que son más vulnerables las personas que viven en zonas de riesgo, en la pobreza, sin acceso a la educación, salud y recursos productivos.

En cuanto a las características del stock edilicio (analizado a partir de una categoría síntesis, como construcción suntuaria, confortable, buena, económica y precaria), predominan las construcciones económicas y precarias. El 60\% de las unidades son económicas y $21 \%$ son precarias. Solo el $12 \%$ de las viviendas son buenas y $4 \%$ confortables, y se encuentran en su mayoría en la zona Puerto o en zonas con periodos de retorno altos.

El 13\% de los hogares presenta hacinamiento, concentrándose básicamente en la zona Norte. En cuanto al nivel educativo, 20\% de los mayores de 18 años no 
terminó la educación primaria. Se reconoce también en este indicador diferencias importantes entre la zona Puerto y las otras.

En cuanto al nivel de servicios, escasas vivienda en la zona tienen saneamiento por red y son muy pocas aquellas que, teniendo sistemas estáticos (pozos negros), utilizan el servicio de recolección ("servicio de barométrica") con asiduidad, lo que da cuenta de que se trata de pozos filtrantes que no cumplen con las normas de salubridad.

Por otra parte, se reconoce también una vulnerabilidad social y organizativa, en tanto son más vulnerables las personas marginadas de los sistemas económicos, políticos y sociales, y las comunidades con instituciones ineficaces y sociedades sin redes de solidaridad.

El 52\% de la población mayor de 18 años cuenta con algún tipo de trabajo. De ellos, el $55 \%$ posee trabajo estable, el $18 \%$ zafral y el $23 \%$ realiza algún tipo de trabajo transitorio ("changas"). Los desocupados propiamente dichos representan el 12\% de los mayores de 18 años.

El 65\% de quienes contestan la pregunta sobre participación en redes u organizaciones sociales declara hacerlo en alguna medida.

Estos aspectos evidencian que si bien el nivel de vulnerabilidad es alto, la zona presenta niveles de participación e inserción en el mundo laboral superiores a las registradas en situaciones similares, como la ciudad de Artigas (también ubicada en el norte del país y con problemas de inundación).

Este perfil, que vincula a la población con el trabajo y con organizaciones, es una información importante a la hora de definir las estrategias tanto de relocalización como de mitigación del evento.

Por último, la vulnerabilidad motivacional y actitudinal da cuenta de que son más vulnerables las personas y comunidades con actitudes fatalistas, barreras culturales y religiosas desfavorables al cambio y a la participación.

Si analizamos la percepción en relación con el evento, es interesante señalar que si bien la represa es vista como la causa principal de las inundaciones tanto en la zona Norte como en la Sur, la opción de que la inundación es "una fatalidad del destino" supera el $12 \%$ en estos dos sectores, mientras que en la zona Puerto apenas supera el $1 \%$.

En la percepción de la población en relación con la zona, se reconocen diferencias en los indicadores analizados. Mientras que en la zona Puerto solo $4 \%$ plantea que es un mal lugar para vivir, en la zona de relocalización, 32\% lo considera. El $80 \%$ de la zona Puerto nunca manejó la posibilidad de irse, mientras que en las zonas Sur y Norte, con mayor frecuencia de inundación, el 52\% consideró esa posibilidad. El 60\% de los entrevistados en estas últimas zonas plantea que se mudarían, mientras que en la zona Puerto solo lo haría el 24\%.

\section{Análisis del impacto de las inundaciones}

Las inundaciones provocan afectaciones a las poblaciones que viven en el área y a la ciudad en general. Desde el punto de vista económico, existen costos directos e indirectos asociados a los bienes muebles e inmuebles perdidos, a la atención a la 
emergencia, entre otros aspectos. Tales costos involucran a muchas instituciones, públicas y privadas, locales y nacionales. También existen pérdidas intangibles, difíciles de ser cuantificadas, pero que muchas veces definen la vida de las personas que habitan la zona inundable.

Este capítulo se concentra en particular en la metodología utilizada para determinar la afectación de las inundaciones en el valor de los bienes inmuebles en suelo privado, aspecto clave en el diseño de herramientas generalizables para determinar pérdidas de manera ajustada en eventos de baja y media intensidad.

\section{Montos de pérdidas estimadas en un periodo de cien años}

El monto de las pérdidas se clasifica en dos grandes ítems: la pérdida del valor del bien por su ubicación en área inundable, entendido como el impacto sobre el valor del barrio; y por otro lado, la afectación directa del evento sobre el predio y sobre el bien inmueble.

El impacto sobre el valor del barrio se define a partir de la consideración de que hay factores que modifican el valor del bien sin que el agua toque siquiera el terreno. Entre ellos se destacan: su condición de predio en zona inundable; la accesibilidad al mismo, dificultada o imposibilitada al momento del evento; la pérdida de calidad ambiental durante el mismo, evidenciada por la invasión de animales desplazados por el agua de su hábitat natural, la presencia de olores, etcétera.

El impacto sobre terreno y mejoras se refiere específicamente a la afectación del bien por el ingreso del agua al predio. El mismo se establece en función del periodo de retorno del evento para cada uno de los predios y de la altura a la que llega el agua. A partir de esto se definen rangos de afectación que se corresponden con valores de reparación, expresados en porcentaje del valor total de la mejora.

\section{Valor del bien}

El valor de las propiedades en la zona se determina a partir de la suma del valor de las mejoras más el valor del terreno.

Para la definición del valor del terreno, se partió de la información proporcionada por la Dirección Nacional de Catastro, que fijó en el año 1997, con vigencia legal en 1999, el Valor Real Territorial (VRT), actualizándolo a través de un índice anual. Este valor original fue calculado sobre la base de un precio por metro cuadrado que considera la acera, la calle a la cual es frentista y la relación "frente-fondo", aplicando al área total un factor de corrección.

Si bien estos valores reales no indican los valores de venta actuales de los terrenos, se puede utilizar la relación que entre ellos existe para aplicar masivamente un factor de corrección a partir de precios unitarios puntuales que se conozcan en la actualidad. A partir de entrevistas a informantes calificados, se identificó este valor "de mercado" para los terrenos de la zona.

Para valorar las mejoras, se determinaron sectores de la vivienda definidos por homogeneidades en el año de construcción, características constructivas, contigüi- 
dad física y significación relativa en relación con la unidad de relevamiento. Para cada uno de ellos, se relevó el nivel de piso terminado con respecto a la calle, la fecha aproximada de construcción, el máximo nivel alcanzado por el agua, los metros cuadrados construidos, los materiales de construcción predominantes, el estado y categoría de la vivienda (Figura 4).

También se relevaron, cuando existían, las patologías en piso, muros y techo: humedades, goteras, grietas, peligro de derrumbe, instalaciones en mal estado, escasa ventilación o iluminación, entre otros.

\section{FIGURA 4 Categoría de viviendas}
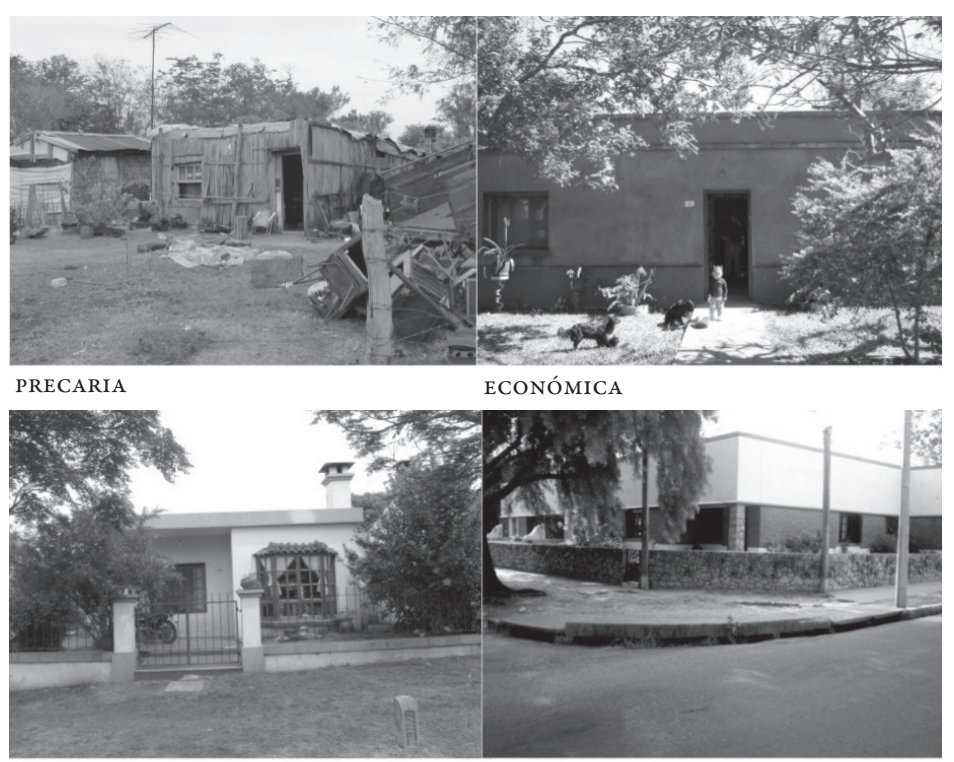

BUENA CONFORTABLE

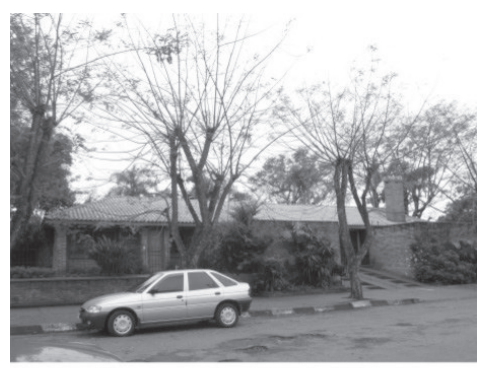

SUNTUOSA

FUENTE ELABORACIÓN PROPIA.

Para su tasación se utilizó el método del "costo por reposición física", que se basa en el principio de sustitución, el que señala que el valor de un inmueble es 
equivalente al de otro de similares características sustitutivo de aquel. Se calcula el valor que representaría construir una edificación nueva con las mismas características de la que se analiza, de manera de obtener el llamado valor de reposición a nuevo (VRN), deduciendo de dicho valor las cantidades cuantificables que representan la pérdida de determinadas condiciones constructivas y funcionales, con el objetivo de obtener el valor de la edificación en su estado real al momento de la tasación, o sea, su valor neto de reposición (VNR).

\section{Afectación del bien por el ingreso del agua al predio}

El impacto de la inundación en cada predio y la posible afectación sobre el bien inmueble se establece en función del periodo de retorno del evento en el mismo y de la altura a la que llega el agua. A partir de esto se establecen rangos de afectación.

Con relación al periodo de retorno, el informe del sistema hídrico da cuenta de que en la ciudad de Bella Unión las curvas de inundación pueden asimilarse a las curvas de nivel, ya que el intervalo de confianza es mayor que la diferencia entre la marca de agua dejada por la inundación ("pelo de agua") y la curva de nivel. Esto permite asociar los impactos a estas curvas. Se asocia entonces a cada curva de nivel una probabilidad de impactos de determinada magnitud, midiendo la magnitud en altura del agua en la vivienda.

A los efectos de este trabajo, se establecieron tres rangos de afectación de las mejoras según la altura que puede alcanzar el agua dentro del predio. Se tomó como altura diferencial los $80 \mathrm{~cm}$ en función de la significación de las afectaciones a la vivienda. ${ }^{6} \mathrm{El}$ rango 1 , afectación del terreno, asume impactos sobre la caminería, espacios exteriores, equipamientos, pozos negros, entre otros. El rango 2, menos de 80 $\mathrm{cm}$, implica la afectación de pisos, zócalos, instalación eléctrica, puertas, revoques, pintura, muebles bajo mesada; en tanto el rango 3, mayor a $80 \mathrm{~cm}$, incorpora a lo anterior ventanas, cielorrasos, techos, sanitaria, estabilidad estructural, entre otros.

Para cada una de las viviendas se analizó la probabilidad de que el agua llegue por encima de los $80 \mathrm{~cm}$ o por debajo de $80 \mathrm{~cm}$ en un periodo de cien años, realizándose una estimación de costo. Este valor se ponderó según la categoría de la vivienda. Para las viviendas en área de relocalización, se tomó el 100\% del valor del bien, equivalente a la expropiación del mismo debido a la incompatibilidad total entre el uso residencial y la inundación en estos periodos de retorno.

\section{Impactos declarados en las encuestas}

A partir de las preguntas realizadas, se pudo saber si la vivienda o el terreno se inundaron alguna vez, en qué años sucedió y cuál fue la altura máxima alcanzada por el agua en la vivienda, medida desde el nivel de piso terminado de la misma. Se obtu-

6 Existen otras categorías para definir rangos en las alturas del agua dentro de la vivienda. En estudios anteriores, este equipo aproximó los rangos a las alturas de los objetos de uso cotidiano (camas, mesas, etcétera), para una mejor comprensión por la población afectada. 
vo, además, información complementaria: la altura de la vivienda en relación con el nivel de calle y desde qué año vivía el encuestado en ella. De esta manera fue posible determinar si la altura declarada por el encuestado se correspondía con la del mayor evento de inundación registrado. En general, la memoria de la gente en relación con los impactos de los últimos treinta años es buena, registrándose diferencias de uno o dos años con respecto al registro de los eventos reales de gran magnitud. Respecto de la altura alcanzada en la vivienda, se asocia a referencias concretas (antepecho, revestimiento, etcétera), lo que permite cierto nivel de fiabilidad.

Esta información permite "validar" los datos de cada una de las unidades de relevamiento, como se grafica en la Figura 5.

FIGURA 5 Altura del agua según encuesta realizada: detalle de manzana 42

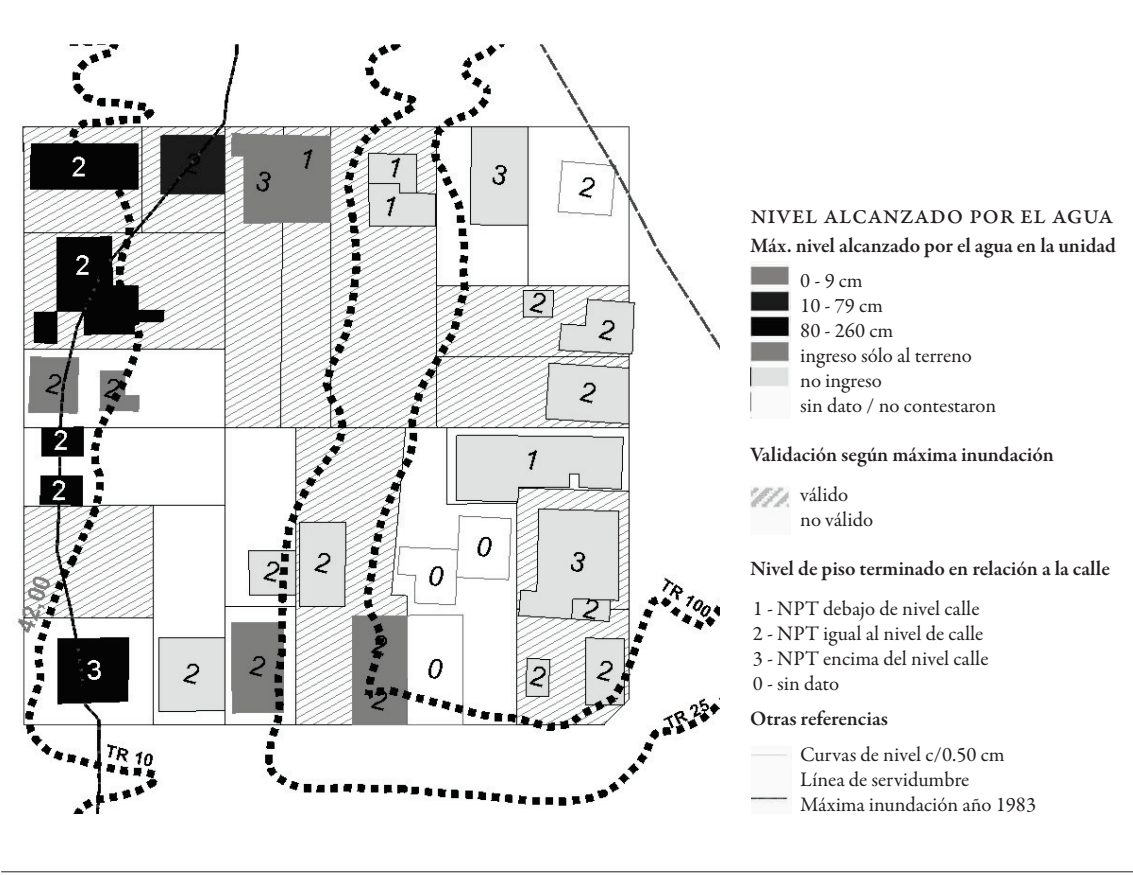

FUENTE ELABORACIÓN PROPIA

\section{Propuestas para el área inundable de Bella Unión}

Como resultados tendientes a la gestión en el mediano y largo plazo de las áreas inundables de la ciudad de Bella Unión, se propone una primera aproximación a medidas de ordenamiento territorial, que, articuladas, promuevan una gestión socioterritorial sustentable. Para ello la coordinación interinstitucional es un factor ineludible. Se presentan en particular tres ítems: los aportes al Plan Local, el pago de las indemnizaciones y el Plan de Relocalizaciones. 


\section{La coordinación interinstitucional como eje}

La investigación realizada pretende asesorar no solo en la elaboración de los productos, sino también en el diseño de los procesos. En particular, se identifican las instituciones involucradas en el territorio en cuestión, explicitando sus competencias y sus prioridades, de manera que las mismas acuerden acciones comunes.

Del análisis surge la existencia de voluntad política de coordinación interinstitucional. En particular se destaca el Plan de Desarrollo Social 2011-2015, Departamento de Artigas (Consejo Nacional de Políticas Sociales, 2010), para la definición de prioridades y coordinación de acciones en el departamento de Artigas que involucra, entre otros, al Ministerio de Vivienda, Ordenamiento Territorial y Medio Ambiente y al Ministerio de Desarrollo Social. En este documento de coordinación de políticas públicas se reconoce como una de las prioridades, la actuación en áreas inundables.

Por otra parte, la coordinación interinstitucional permite replicar en situaciones similares las experiencias aprendidas ya sea tanto por éxitos como por fracasos.

La voluntad de coordinar las indemnizaciones con el Plan de Relocalizaciones liderado por la Dirección Nacional de Vivienda es una señal positiva, que permite pensar en una operativización de medidas en cortos plazos.

\section{Aportes al Plan Local de la ciudad}

La Ley de Ordenamiento Territorial y Desarrollo Sostenible ( $\left.\mathrm{N}^{\circ} 18.308\right)$ define como uno de sus instrumentos el Plan Local de Ordenamiento Territorial y Desarrollo Sostenible. La elaboración y aprobación de este instrumento es de competencia exclusiva de los gobiernos departamentales. Los organismos del gobierno nacional colaboran en su elaboración y en la coordinación con los instrumentos nacionales

El Plan Local contribuye a planificar el desarrollo integrado y ambientalmente sustentable del territorio, mediante el ordenamiento, la transformación y el control de la utilización del suelo, incluyendo la definición de una estrategia de utilización del territorio, coherente con los instrumentos de ordenamiento territorial de ámbito superior, la categorización del suelo, su ordenamiento estructural y los lineamientos para otros instrumentos de planificación.

En la actualidad no existe un Plan Local para la ciudad de Bella Unión, estando prevista su elaboración para el presente quinquenio de gobierno. Este trabajo pretende contribuir en el proceso planificador que se está iniciando, en particular en tres aspectos: elaboración del mapa de riesgo, que coordinan las Intendencias Departamentales con la Dinagua, la categorización del suelo, que se coordina con la Dirección Nacional de Ordenamiento Territorial (Dinot) y la definición de proyectos urbanos ribereños asociados a las acciones que corresponde emprender a los Municipios.

El mapa de riesgo es un instrumento de planificación y gestión que permite definir las acciones específicas según los niveles de riesgo. El proyecto de Directrices de Inundaciones de Ribera plantea que "todo centro poblado situado en la ribera de un curso de agua deberá cartografiar sus zonas de riesgo por inundaciones 
considerando los niveles de amenaza y vulnerabilidad". A partir de tal instrumento deberán definirse las medidas de previsión, prevención y mitigación. El mapa de riesgo requiere ser actualizado, en particular a partir de nuevos estudios del sistema hídrico en función de la variabilidad y el cambio climático. La actualización podrá realizarse conjuntamente con la del Plan Local, o cuando se reconozca un cambio importante en las dinámicas o metodologías de análisis utilizadas. Este trabajo da elementos para la definición de dicho mapa.

A efectos de aportar hacia la categorización del suelo urbano, se realizó una primera zonificación del área urbana inundable de la ciudad de Bella Unión a partir de su caracterización urbano-territorial y de las diferentes afectaciones del evento de inundación. Se definieron tres zonas de actuación en función de sus características intrínsecas y su relación con el sistema hídrico. La zona Puerto se presenta como el sector más consolidado, estructurándose sobre el eje de las principales vías urbanas de la ciudad (calles Rivera y Artigas). Presenta viviendas en general buenas que cuentan con servicios; la población es estable y vive en la zona desde hace años, con un fuerte arraigo al barrio. Las medidas previstas para esta zona jerarquizan estrategias de mitigación, en particular normativas especiales en la cual se consideren especificaciones en las construcciones (por ejemplo, altura determinada de instalaciones, construcciones elevadas, sistema de saneamiento adecuado, etcétera). En las zonas Sur y Norte predominan viviendas económicas. Se encuentran más alejadas del centro y poseen mayores carencias de equipamientos urbanos. En los sectores de mayor proximidad al río se definieron subzonas asociadas a estrategias de relocalización.

Tomando en cuenta las características particulares de cada sector, se plantea el posterior estudio de una estrategia específica que integre aspectos constructivos de las viviendas y acciones en el espacio público.

La programación y proyecto de los espacios del río es fundamental para la calificación de las áreas inundables. El lago de la represa redujo sensiblemente el área de parque, mientras que las expropiaciones generarán nuevo suelo vacante en zonas alejadas del espacio habitual de uso de la ciudad, que es necesario incorporar al espacio público. La recuperación de la naturalidad del espacio del río, incorporándolo al imaginario colectivo, debe ser una prioridad, considerando la sostenibilidad (costos de mantenimiento y propuesta de gestión) de los espacios públicos que se propone conformar en las soluciones que se desarrollen.

\section{Indemnizaciones por impacto de la construcción de la represa}

De los montos estimados como pérdidas por eventos de inundación, se detectó qué porcentaje corresponde al impacto de la presa, a partir de los estudios hidráulicos realizados. Se identificaron zonas que han pasado de área no inundable a inundable, zonas que aumentaron el periodo de retorno y viviendas que han visto incrementada la afectación directa del agua (aumento en la altura del agua en la vivienda). En las zonas de riesgo alto se sugiere la expropiación, por lo que el costo total se compone del monto de indemnización y del costo de una vivienda digna en función de 
las familias que ocupan este sector. Esto da elementos a los efectos de coordinar con las políticas públicas de vivienda. En las zonas de riesgo medio, los costos se discriminan según el valor de afectación de la represa y el valor total de la inundación.

Las zonas de riesgo alto se consideran zonas incompatibles con el uso habitacional, por lo que se propone su definición como zona de relocalización.

Del análisis de la zona se reconoce la existencia de hogares con diversas situaciones, que van desde familias con viviendas económicas que viven desde hace años en la zona, hasta hogares que viven en situaciones precarias tanto social como habitacionalmente. En esta zona, las viviendas son en su mayoría precarias (el 45\%) y el resto, casi todas económicas.

Desde este trabajo se aportan dos elementos para la definición de las políticas de vivienda: el costo estimado de la operación de relocalización y el perfil de las familias que componen el universo de posibles realojados.

En cuanto al costo, en la zona de relocalización se encuentran 81 viviendas ( 37 precarias, 34 económicas, 8 buenas y 2 confortables), cuyo valor de expropiación se estima en 1.000.000 dólares ( sin incluir precio de suelo). El costo de la vivienda de relocalización es superior al de la expropiación, por lo que debería agregarse un costo de reposición estimado en 2.100 .000 dólares, con un costo de terreno estimado en 5.000 dólares por unidad. A estos costos es necesario integrar los propios de la urbanización, indispensables para operativizar la solución.

En cuanto al perfil social, además del procesamiento de la información para la investigación, se generó una base de datos previendo su vinculación con las de otros organismos públicos responsables de las políticas sociales, como el Ministerio de Desarrollo Social. Esto permitirá potenciar el uso de la información generada en instancias posteriores de apoyo al proceso de relocalización.

\section{Consideraciones finales}

Más allá de reconocer las particularidades urbanas y sociales de la situación local, este trabajo, en tanto parte de un proceso de investigación en gestión del riesgo, se propone contribuir al reconocimiento de las invariantes y particularidades de las áreas inundables de ribera en zona de llanura, permitiendo mejorar el análisis de otros casos nacionales y de la región, y dando nuevos elementos para la mejora de la caja de herramientas para la actuación en el territorio.

En los últimos cincuenta años han sido múltiples las propuestas de intervención en áreas inundables en nuestro país. Su puesta en práctica ha dejado a la luz una serie de problemas recurrentes en distintos momentos históricos y lugares geográficos. Los trabajos realizados por el equipo han permitido identificar y caracterizar algunos de ellos: falta de control de las zonas inundables luego de efectivizados los realojos, con la generación de nuevas ocupaciones posteriores; relocalización de las familias en condiciones de hábitat incluso más precarias que las del área inundable; pérdida de redes sociales y medios de subsistencia, que trae aparejados nuevos conflictos sociales; expansiones urbanas innecesarias sobre suelo rural y muchas veces 
incluso en zonas de riesgo, entre otros muchos aspectos.

Para revertir estas tendencias, la planificación de las áreas inundables requiere a la vez profundizar en las metodologías de abordaje de cada uno de los componentes del riesgo (amenaza y vulnerabilidad) e incorporar la gestión del riesgo en el ordenamiento territorial.

Se toma como posicionamiento de partida la adopción de medidas tendientes a la más alta naturalidad posible. Estudios anteriores en situaciones similares ${ }^{7}$ dan cuenta de que la opción por soluciones estructurales implica costos económicos y ambientales que no son justificables solo con el objetivo de controlar inundaciones de este nivel de impacto.

Trabajar en este sentido implica ajustes en los estudios desde la modelación del sistema hídrico (mejora de la información hidrológica, pluviométrica y topográfica, la utilización de nuevos modelos que aproximen mejor a la dinámica del cuerpo de agua). En este caso en particular, el ajuste de las curvas de nivel de 2 a 0,5 metros y la verificación de los resultados del modelo con una encuesta permiten un avance que habilita la aplicación más ajustada de medidas no estructurales.

En cuanto a la vulnerabilidad, se realizaron ajustes en la metodología utilizada, los cuales permiten análisis comparativos con otras situaciones urbanas en los que se considera las diferentes dimensiones de la vulnerabilidad: los aspectos físicomateriales, los socio-organizativos y los motivacional-actitudinales.

Por otro lado, se profundizó en la caracterización de las construcciones, lo que habilita una aproximación mayor a la valoración económica del stock edilicio y mejorar el cálculo de depreciación de los sucesivos eventos adversos. Esta metodología de valoración puede ser replicada en situaciones similares (nacionales o regionales), ajustando según costos locales de construcción. Asimismo, genera insumos para la implementación de seguros contra este tipo de eventos, política escasamente desarrollada en nuestro país, salvo para algunos eventos adversos para la producción agropecuaria (granizo, por ejemplo).

La metodología presentada saca ventaja de la actuación a pequeña escala, habilitada por la magnitud de los eventos. Se aproxima así a un análisis interescalar e interdisciplinario que atiende diferentes dimensiones del problema, apuntando al diseño de estrategias y acciones particularizadas e integrales. Cada componente podrá ser desarrollado en futuros trabajos, verificando, ajustando y validando la metodología. OEURE

En particular, trabajos del Instituto de Mecánica de los Fluidos e Ingeniería Ambiental de la Facultad de Ingeniería en las ciudades de Tacuarembó y San Carlos, y de la Dirección Nacional de Aguas en Treinta y Tres. 


\section{Referencias bibliográfcas}

Barrenechea, J., Gentile, E., González, S. \& Natenzon, C. (2000). Una propuesta metodológica para el estudio de la vulnerabilidad social en el marco de la teoría social del riesgo. Ponencia presentada en las IVas Jornadas de Sociología, Facultad de Ciencias Sociales, Universidad de Buenos Aires. Buenos Aires, 6 al 10 de noviembre de 2000.

Cardona, O. (2001). Estimación holística del riesgo sísmico utilizando sistemas dinámicos complejos. Tesis doctoral. Universitat Politécnica de Catalunya, España.

Cardona, O. (2003). La necesidad de repensar de manera holistica los conceptos de vulnerabilidad y riesgo. Una crítica y una revisión necesaria para la gestión. Bogotá: Centro de Estudios sobre Desastres y Riesgos (Cederi), Universidad de los Andes. http://www.desenredando.org/ public/articulos/2003/rmhcvr/rmhcvr_may-08-2003.pdf

Comisión Técnica Mixta de Salto Grande. (2011). Cotas de inundación en Bella Unión y Pueblo Cuareim. Informe de trabajo. Manuscrito no publicado.

Consejo Nacional de Políticas Sociales. (2010). Plan de Desarrollo Social, 2011-2015. Departamento de Artigas. Manuscrito no publicado.

Estrategia Internacional para la Reducción de Desastres (EIRD). (2005). Marco de acción de Hyogo para 2005-2015: Aumento de la resiliencia de las naciones y las comunidades ante los desastres. Conferencia Mundial sobre la Reducción de los Desastres, 18 a 22 de enero de 2005, Kobe, Hyogo, Japón. http://www.comunidadandina.org/predecan/doc/ext/hyogo.pdf

Estrategia Internacional para la Reducción de Desastres (EIRD), Naciones Unidas, Unidad Regional para las Américas. (2004). Terminología: Términos principales relativos a la reducción del riesgo de desastres. http://www.eird.org/esp/terminologia-esp.htm, Actualizado 31-03-04

Estrategia Internacional para la Reducción de Desastres (EIRD), Naciones Unidas, Unidad Regional para las Américas. (2008). La gestión del riesgo de desastres hoy, contextos globales herramientas locales. Versión electrónica en http://eird.org/gestion-del-riesgo/index.html

Grupo de Gestión Integral del Riesgo. (2010). Inundación 2009: Relevamiento Artigas. Informe preliminar. Manuscrito no publicado.

Instituto de Teoría y Urbanismo (ITU). (2007). Relevamiento del área inundable de la ciudad de Treinta y Tres. Manuscrito no publicado.

Lavell, A. (1996). Degradación ambiental, riesgo y desastre urbano. Problemas y conceptos: Hacia la definición de una agenda de investigación. En M. A. Fernández (Comp.), Ciudades en riesgo. Degradación ambiental, riesgos urbanos y desastres (pp. 21-60). Lima: Red de Estudios Sociales de Prevención de Desastres de América Latina (La Red).

Lavell, A. (2003). La gestión local del riesgo. Nociones y precisiones en torno al concepto y la práctica. Panamá: Centro de Coordinación para la Prevención de los Desastres Naturales en América Central (Cepredenac), Programa de Naciones Unidas para el Desarrollo (PNUD).

Lavell, A. (2002) Sobre la gestión del riesgo: apuntes hacia una definición. http://es.scribd.com/ doc/91381522/riesgo-apuntes

Ministerio de Vivienda, Ordenamiento Territorial y Medio Ambiente (Mvotma), Dirección Nacional de Aguas. (2011). Proyecto de Directrices de Inundaciones de Ribera. (http://www.mvotma.gub.uy/dinasa/index.php?option=com_content\&view=article\&id=161 -3 de marzo 2011). 
Ministerio de Vivienda, Ordenamiento Territorial y Medio Ambiente (Mvotma), Dirección Nacional de Vivienda. (2010). Mi lugar, entre todos. Sistema Público de Vivienda de Interés Social 2010-2014. Montevideo: Autor.

Natenzon, C. (1995). Catástrofes naturales, riesgo e incertidumbre. Buenos Aires: Facultad Latinoamericana de Ciencias Sociales, Flacso-Argentina.

Piperno, A. \& Sierra, P. (2009). Metodologias para la planificación y gestión de áreas urbanas inundables. $I+D$ 2006. Manuscrito no publicado.

Piperno, A., Sierra, P., Varela, A. \& Failache, N. (2009). Inundaciones urbanas en el Uruguay, Montevideo: Tradinco.

Uruguay. Ley $N^{o} 15845$ (1986). Servidumbre administrativa de ocupación temporaria de aguas por la represa de Salto Grande.

Uruguay. Ley $N^{o} 18308$ (2008). Ley de Ordenamiento Territorial y Desarrollo Sostenible. 